\title{
Analysis of Time to Form Colony Units for Connective Tissue Progenitor Cells (Stem Cells) Harvested From Concentrated Bone Marrow Aspirate and Subacromial Bursa Tissue in Patients Undergoing Rotator Cuff Repair
}

\author{
Arthur Landry, B.A., Benjamin J. Levy, M.D., Mary Beth McCarthy, B.S., \\ Lukas N. Muench, M.D., Colin Uyeki, B.A., Daniel P. Berthold, M.D., Mark P. Cote, D.P.T., \\ and Augustus D. Mazzocca, M.D.
}

\begin{abstract}
Purpose: To evaluate the time required for colonies to develop from concentrated bone marrow aspirate (cBMA) and subacromial bursal tissue samples. Methods: Samples of cBMA and subacromial bursa tissue were harvested from patients undergoing rotator cuff repair surgery between November 2014 and December 2019. Samples were analyzed for time to form colonies and number of colonies formed. The impact of age, sex, and cellularity (cBMA only) was analyzed. Samples were cultured and evaluated daily for colony formation in accordance with the guidelines of the International Society for Cellular Therapy. Demographic factors were analyzed for impact on time to form colonies and number of colonies formed. Results: Samples of cBMA were obtained from 92 patients. Subacromial bursa tissue was obtained from 54 patients. For cBMA, older age was associated with more days to form colonies $(P=.003)$, but sex $(P=.955)$ and cellularity $(P=.623)$ were not. For bursa, increased age was associated with longer time to form colonies $(P=.002)$ but not sex $(P=.804)$. Conclusions: Increased age (in cBMA and subacromial bursa tissue) and lower initial cellularity (in cBMA) are associated with longer time to form colonies in culture. Clinical Relevance: Although connective tissue progenitor cells are widely used in orthopaedic practice, there are few metrics to determine their efficacy. Time to form colonies may serve as an important measurement for determining connective tissue progenitor cell viability for augmentation of rotator cuff repair. Subacromial bursa tissue may represent a viable alternative to cBMA for augmentation of rotator cuff repair, capable of forming colonies expediently in vivo.
\end{abstract}

From the University of Connecticut School of Medicine (A.L.) and Department of Orthopaedic Surgery, UConn Health (B.J.L., M.B.M., L.N.M., C.U., D.P.B., M.P.C., A.D.M.), Farmington, Connecticut, U.S.A.; and Department of Orthopaedic Sports Medicine, Technical University of Munich, Germany (L.N.M., D.P.B.)

The authors report the following potential conflicts of interest or sources of funding: M.P.C. reports personal fees from Arthroscopy Association of North America (AANA), outside the submitted work. A.D.M. reports personal fees and nonfinancial support from Arthrex, outside the submitted work. Full ICMJE author disclosure forms are available for this article online, as supplementary material.

Received March 6, 2020; accepted July 16, 2020

Address correspondence to Benjamin J. Levy, M.D., Department of Orthopaedics, UConn Health, 263 Farmington Ave, Farmington, CT 06030-4037. E-mail:levyben88@gmail.com or blevy@uchc.edu

(C) 2020 THE AUTHORS. Published by Elsevier Inc. on behalf of the Arthroscopy Association of North America. This is an open access article under the CC BY-NC-ND license (http://creativecommons.org/licenses/by-nc-nd/4.0/).

2666-061X/20283

https://doi.org/10.1016/j.asmr.2020.07.013
G iven the difficulty in producing reliable healing in patients undergoing rotator cuff repair, there has been an increase in surgeon application of connective tissue progenitor (CTP) cells to repaired tendons intraoperatively. ${ }^{1,2}$ CTPs ultimately differentiate based on their surroundings, capable of becoming skeletal muscle cells, or tenocytes, potentially aiding tendon healing. ${ }^{3-5}$ Recently, there has been further discussion regarding the nomenclature of these cells. Given their limited regenerative capacity, these cells should be referred to as "connective tissue progenitor" cells, rather than mesenchymal stem cells. ${ }^{6}$ For consistency, the pluripotent cells discussed for the remainder of this study will be referred to as "CTPs." Intraoperative use of these cells has been described extensively via the harvesting of concentrated bone marrow aspirate (cBMA) for applications in orthopaedic surgery. ${ }^{1,2,7-9}$ Donor sites include the anterior and posterior iliac crest, proximal 
humerus, and distal femur. ${ }^{2,7,10-12}$ Additional work has discussed the presence of these cells in subacromial bursa tissue as well. ${ }^{13-17}$ These studies call into question the routine debridement and disposal of this tissue. ${ }^{16,18}$

Although the adjuvant use of CTPs has become more common, little is known about potential factors that may impact the quality, quantity, or healing potential of these cells. ${ }^{9}$ One of the main determinants differentiating CTPs from other cells is their ability to form colonies. ${ }^{19}$ Colonies are measured in terms of colonyforming units (CFUs), indicating the nature of these cells to form colonies from a single cell. ${ }^{20}$ CTP expression of specific surface markers (e.g., CD105, 73, 90), and their ability to differentiate separates these cells from other cells in cBMA or bursa. ${ }^{21}$ Once a group of cells has formed in a unit together (8 cells), one colony has formed. ${ }^{21-23}$ The number of CFU fibroblasts per volume in a given sample of harvested CTPs has been described as a measurable factor impacting CTP "quality" previously in the orthopaedic literature. ${ }^{24}$ Although CFU count has been described as a metric to assess CTPs, there is still significant debate regarding optimal in vitro determinants of in vivo CTP viability.

The speed at which colonies are formed may be a factor in how well they can aid healing tendon. Anecdotally, the time to form colonies has been noted to vary. Guan et al. ${ }^{25}$ have discussed the rate of colony formation as a proxy for efficacy of stem cell growth in umbilical cells. Although the significance of time to colony formation remains unknown, this may represent a useful metric for viability of a particular group of CTPs.

A better understanding of factors associated with the ability of a transplanted sample to produce colonies in vivo is needed to maximize the potential benefit of progenitor cell augmentation of rotator cuff repair. Factors that have the potential to influence colony formation time, such as patient age, sex, and sample cellularity of the acquired samples, were studied. The purpose of this study was to evaluate the time required for colonies to develop from cBMA and subacromial bursal tissue samples. The authors of this study hypothesized that there would be a measurable increase in time to form colonies in samples of cells from older patients and samples with less cellularity.

\section{Methods}

Samples of cBMA and subacromial bursa tissue were harvested from patients undergoing rotator cuff repair surgery between November 2014 and December 2019. This protocol was approved by the institution's institutional review board (\#06-577-2). cBMA samples were collected between November 2014 and March 2019 from consecutive patients with excess cBMA available for analysis. Patients were excluded from collection if cBMA was not harvested or there was not excess cBMA available for laboratory analysis. Bursa samples were collected between January 2019 and December 2019 on consecutive patients with excess bursal tissue available for analysis. Patients without excess bursal tissue available were excluded from collection.

For cBMA procurement, to summarize, a 14-gauge bone marrow aspiration trocar, fit with a $60-\mathrm{mL}$ syringe containing $3 \mathrm{~mL}$ of Anticoagulant Citrate Dextrose Solution A (Baxter Healthcare Corp., Deerfield, IL), was inserted 2.5 to $3 \mathrm{~cm}$ into the medial aspect of the greater tuberosity (Bone Marrow Aspiration Kit; Arthrex, Naples, FL). In a standardized method of aspiration, the operative surgeon pulled back on the syringe to maximize suction, allowing $20 \mathrm{~mL}$ of aspirate to flow into each of 6 syringes for a total of $120 \mathrm{~mL}$ of aspirate. cBMA was concentrated using cell sorting technology with the Arthrex Angel System (Arthrex). Then, $120 \mathrm{~mL}$ of the cBMA was concentrated by centrifugation for 25 minutes. For all cBMA samples, volume (milliliters) and nucleated cell count (million/ $\mathrm{mL}$ of cBMA) were recorded. Samples were plated using 100-mm Primaria dishes (ThermoFisher Scientific, Agawam, MA) with $10 \mathrm{~mL}$ of complete Dulbecco's modified eagle medium (Thermo Fisher Scientific, Waltham, MA), containing $10 \%$ fetal bovine serum and $0.1 \%$ penicillin/streptomycin sulfate. Cells were grown to confluence in a $5 \% \mathrm{CO}_{2}$ incubator at $37^{\circ} \mathrm{C}$. Media was aspirated and replaced every 3 days. Cultures were checked for evidence of colony formation at daily intervals by a trained technician. Guidelines provided by The International Society for Cellular Therapy were used. A colony was defined as a group of 8 or more cells in a culture. ${ }^{21,22}$ CTPs must meet 3 criteria: (1) express surface markers CD 73, CD90, and CD105; (2) differentiate into fat, bone, or cartilage; and (3) adhere to tissue culture plastic. ${ }^{21}$ Once colonies were identified, dishes were systematically examined at $10 \times$ magnification to determine total number of colonies (CFU) present (Fig 1). A single, trained technician scanned one-quarter of each plate and extrapolated to give the total number of colonies formed for each sample. This process was performed on the day colonies were first identified. Expedited counting was necessary, given rapid confluence of colonies making counting difficult after that point.

Subacromial bursa tissue was harvested during routine bursectomy before rotator cuff repair. Bursa samples were harvested with a Blakesley grasper device (Jarit; Integra LifeSciences, Princeton, NJ), and transported from the operating room to the biologics lab in sterile saline in an expedited fashion. Then, $200 \mathrm{mg}$ of tissue (approximately the size of an eraser head) was massed and prepared for culture using the chopping method for each sample, as described by Morikawa et al. ${ }^{26}$ Cultures were grown in the process described 


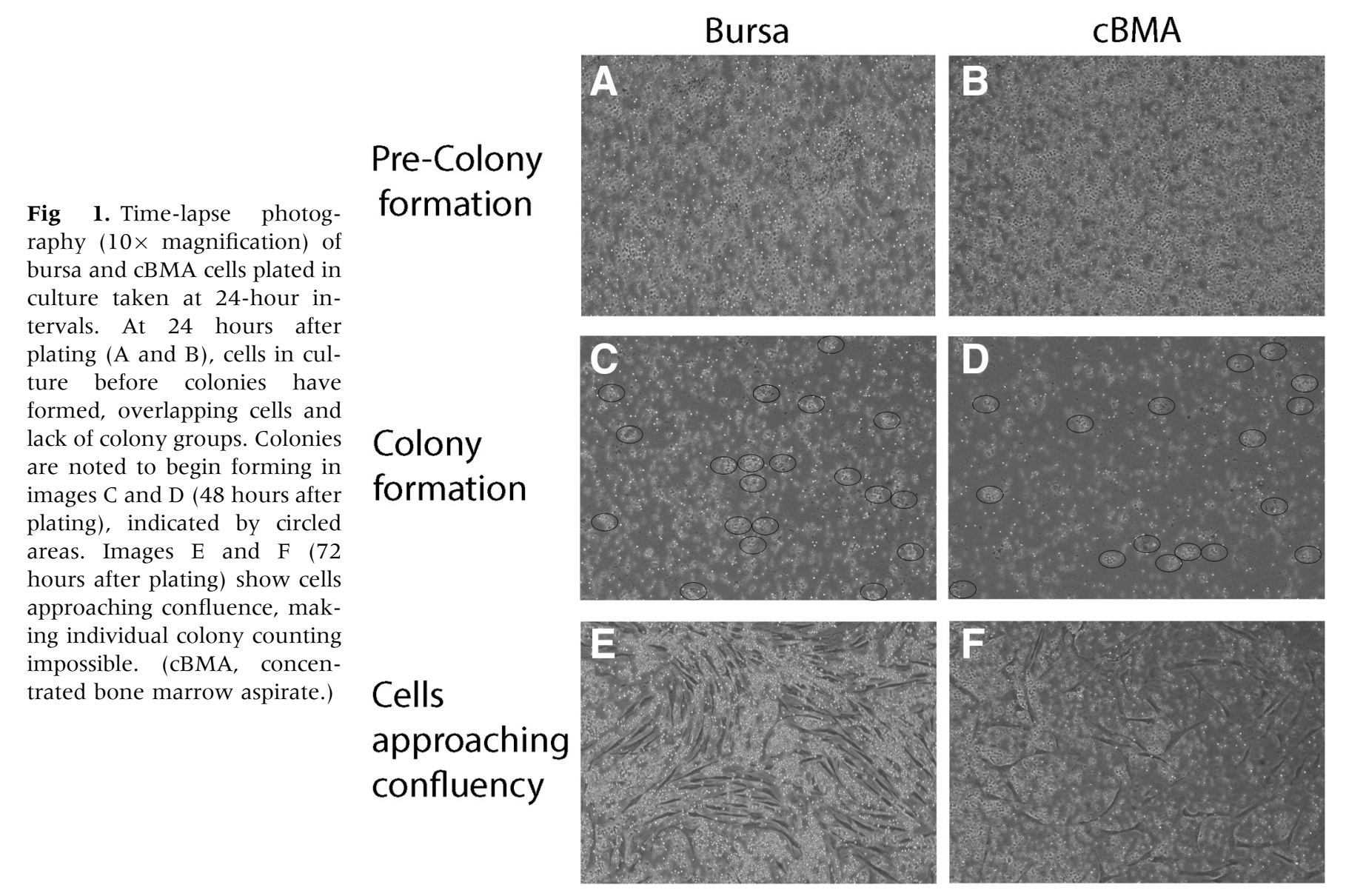

Fig 1. Time-lapse photography (10× magnification) of cBMA cells plated in formed, overlapping cells and lack of colony groups. Colonies are noted to begin forming in images $C$ and D (48 hours afte plating), indicated by circled (t) approaching con (cBMA, concen-

\section{approaching confluency}

previously for cBMA. As with cBMA samples, cultures were checked under a microscope at daily intervals, and total number of colonies recorded.

For all cBMA samples, volume (milliliters) and nucleated cell count (million/mL of cBMA), or "cellularity" were recorded. "Cellularity" data are not available for bursa samples given the method of obtaining "cellularity" measurements, as bursa is a solid structure, rather than liquefied.

Fluorescence-activated cell sorting analysis was performed to assess for surface markers, CD73, CD90, and CD45 to further confirm that the cells obtained from the isolated bone marrow and bursa tissue were CTPs. Cells were grown in complete media, trypsinized in $0.25 \%$ trypsin/ethylenediaminetetraacetic acid at confluence, rinsed, and centrifuged. Cells were labeled with either phycoerythrin or fluorescein isothiocyanate antibodies for CD73, CD90, and CD105 as previously described. ${ }^{21}$ Although there is no specific cell marker for CTPs, it has been shown that cells positive for CD73 and CD90 as well as negative for CD45 are characteristic of CTPs. ${ }^{21,23}$ Cell fluorescence was analyzed (Fig 2) with a FACSCalibur instrument (BD Biosciences, San Jose, CA). Positive and negative controls of each antibody also were analyzed to ensure antibody-specific cell fluorescence.

The data were further analyzed based on sex, age, and cellularity (for cBMA only), to assess for these demographics' impact on time to form colonies or total number of colonies formed. Finally, days required to form colonies was compared to total number of colonies formed for each subset (BMA and bursa).

\section{Statistical Analysis}

Descriptive statistics including mean and standard deviation as well as median and range were calculated to characterize the data. Linear regression was used to examine differences in CFUs, whereas days to form colonies was considered count data and analyzed with Poisson regression. Separate univariate models were constructed to examine differences in days and CFUs according to age, sex, and source. To account for differences in age-related effects, differences days to form colonies between BMA and bursa are presented with adjustment for age. Results are reported as relative ratios with corresponding 95\% confidence intervals (CIs). All analyses were performed using Stata (Stata 


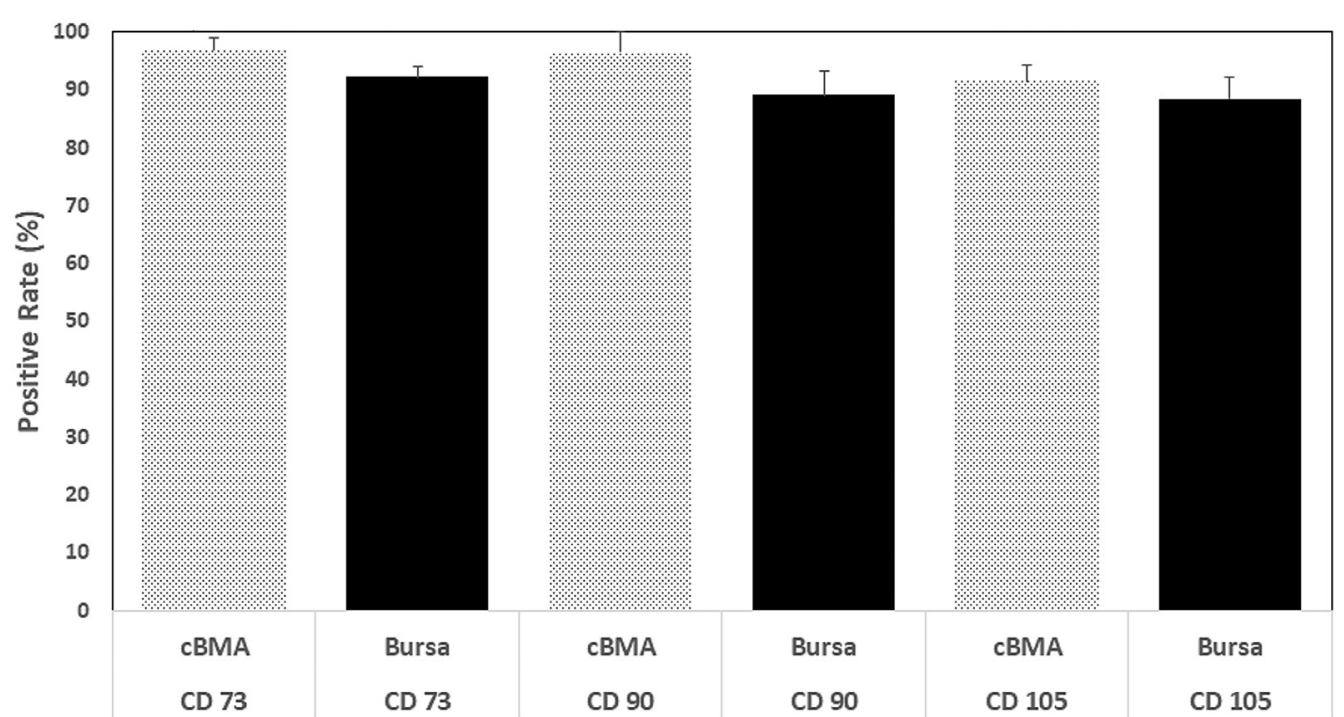

Fig 2. Presence of confirmatory surface markers (CD73, CD90, and CD105) for presence of CTPs in cBMA and subacromial bursa samples. FACS analysis of 8 random cBMA and subacromial bursa samples were performed for the aforementioned analysis. (cBMA, concentrated bone marrow aspirate; FACS, fluorescence-activated cell sorting.)
Statistical Software, Release 15; StataCorp LLC, College Station, TX, 2017).

\section{Results}

The total number of rotator cuff repairs performed at the institution by the operative surgeon from November 2014 to December 2019 was 419.

\section{Fluorescence-Activated Cell Sorting}

CD73 was found to be present in $96.9 \%( \pm 2.1)$ of sampled cBMA and $92.3 \%( \pm 1.8)$ of sampled subacromial bursa tissue cells. CD90 was present in $96.5 \%$ $( \pm 3.4)$ of sampled cBMA and $89.1 \%( \pm 4.1)$ of sampled subacromial bursa tissue cells. CD105 was present in $91.5 \%( \pm 2.9)$ of sampled cBMA and $88.5 \%( \pm 3.8)$ of sampled subacromial bursa tissue cells (Fig 2).

\section{Concentrated Bone Marrow Aspirate}

There were 92 patients included in the cBMA portion of the study. Of these 92 patients, 64 were male (mean age $56.2 \pm 7.0$ years), and 28 were female (mean age $58.9 \pm 5.6$ years). The overall average time to form colonies was $7.1 \pm 1.5$ days $(7.1 \pm 1.5$ days in male patients compared with $7.1 \pm 1.5$ days in female patients; $P=.955,95 \%$ CI 0.86-1.15). The average time to form colonies for cBMA samples in patients younger than 60 years of age was $6.6 \pm 1.5$ days $(\mathrm{n}=56)$ compared with $7.8 \pm 1.2$ days for patients age 60 years or older $(\mathrm{n}=36)$. There was a statistically significant relationship between time to form colonies and age based on a regression model $(P=.003,95 \%$ CI 1.00 $1.02)$, noted in Figure 3 . The mean number of nucleated cells per cBMA sample was 127,606. There was not a significantly significant association of time to form colonies for cBMA samples based on number of nucleated cells present ( $P=.623,95 \%$ CI 1.00-1.00).
The average total number of colonies formed in cBMA samples was $1459 \pm 729 \mathrm{CFU} / \mathrm{mL}$. Total number in male patients was $1576 \pm 774 \mathrm{CFU} / \mathrm{mL}$ compared with $1193 \pm 522 \mathrm{CFU} / \mathrm{mL}$ in female patients $(P=.020$, $95 \%$ CI 61.62-705.1), a statistically significant difference, as noted in Figure 4. This relationship held true in multivariate regression, taking into account the possible impact of cellularity and age $(P=.0027,95 \%$ CI 26.17 422.4). The total colonies formed for patients younger than 60 years of age was $1471 \pm 813 \mathrm{CFU} / \mathrm{mL}(\mathrm{n}=56)$ compared with $1441 \pm 572 \mathrm{CFU} / \mathrm{mL}$ for patients 60 years or older $(\mathrm{n}=36),(P=.655,95 \% \mathrm{CI}-17.51$ to 27.74). There was a significant relationship between initial sample number of nucleated cells and total number of colonies present at time of counting, $(P \leq$ $.001,95 \%$ CI 0.01-0.01). This relationship remained with multivariate analysis, accounting for confounding of sex and age ( $P \leq .001,95 \%$ CI 0.01-0.01).

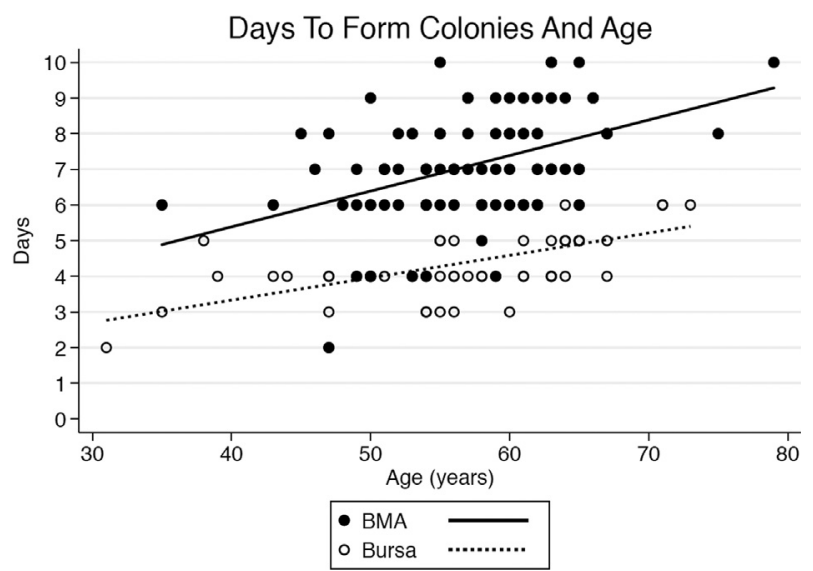

Fig 3. Days to form colonies versus age for cBMA and bursa. (cBMA, concentrated bone marrow aspirate.) 


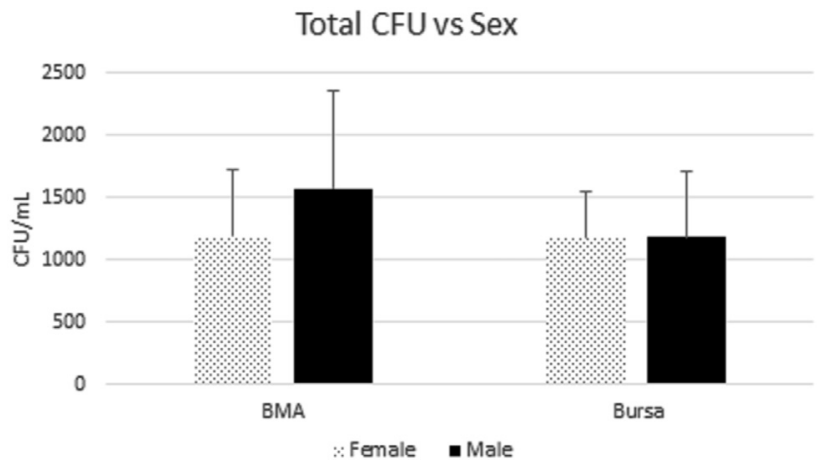

Fig 4. Total CFUs formed for cBMA and bursa. (cBMA, concentrated bone marrow aspirate; CFU, colony-forming units.)

There was no statistical relationship found between days to form colonies and total number of colonies formed for cBMA samples, $(P=.586,95 \%$ CI 1.00 $1.00)$.

\section{Subacromial Bursa Tissue}

There were 54 patients included in the subacromial bursa tissue collection portion of the study, all 54 had data available regarding time to form colonies. In total, 51 of these patients had total colony number formed data available for analysis ( 3 patients were erroneously not included in quantification of colony number formed). Of the 54 total patients, 38 were male (mean age $57.8 \pm 9.2$ years) and 16 were female (mean age $55.6 \pm 8.5$ years). The average time to form colonies overall was $4.4 \pm 1.0$ days $(4.4 \pm 1.0$ days for males and $4.3 \pm 0.9$ days for females) $(P=.804,95 \%$ CI 0.88 1.18 ). For the 28 patients younger the age of 60 years, the average time to form colonies was $3.9 \pm 0.8$ days, and $4.9 \pm 0.8$ days for the 26 patients age 60 years or older ( $P=.0002,95 \%$ CI 1.01-1.02), a relationship that remained apparent based on continuous regression analysis (Fig 3).

There were 51 patients with data available regarding total colonies formed. In total, 35 were male (mean age $58.7 \pm 7.3$ years) and 16 were female (mean age $55.6 \pm$ 8.5 years). The average total number of colonies formed was $1184 \pm 470 \mathrm{CFU} / \mathrm{mL}$ overall, for males, $1183 \pm 515$ $\mathrm{CFU} / \mathrm{mL}$, and $1184 \pm 352 \mathrm{CFU} / \mathrm{mL}$ for the female subgroup ( $P=.993,95 \%$ CI -291.94 to 289.46$)$, as noted in Figure 4.

The average total number of colonies formed for patients younger than 60 years of age $(\mathrm{n}=26)$ was 1347 $\pm 444 \mathrm{CFU} / \mathrm{mL}$ compared with $1013 \pm 434 \mathrm{CFU} / \mathrm{mL}$ in patients 60 years or older $(\mathrm{n}=25),(P=.303,95 \% \mathrm{CI}$ -25.87 to 8.21 ).

There was no statistical relationship found between days to form colonies and total number of colonies formed for bursa samples $(P=.390,95 \% \mathrm{CI}$ $1.00-1.00)$.
The formation of colonies in the cBMA and bursa samples can be viewed in microscopy images used for confirmation of colony formation, noted in Figure 1. A comparison of time (in days) to form colonies and total number of colonies formed (CFU) between bursa and cBMA can be viewed in Figures 5 and 6, respectively.

\section{Discussion}

The results, in terms of time to form colonies, support the literature that increased patient age (in both bursa and (BMA), but not sex of patient or cellularity of sample, increases time to colony formation of CTPs. This study presents time to form colonies in culture as a novel metric for measuring CTP quality. The clinical implications of these results must be supported by future in vivo analyses. Further, the work presented supports that bursal-derived progenitor cells may represent an effective method of CTP augmentation during routine rotator cuff repair.

Increased age was noted to be associated with increased time to form colonies in both cBMA and subacromial bursa tissue. Unexpectedly, male sex was associated with greater number of total colonies formed in cBMA but not subacromial bursa tissue. More work must be done to understand the significance of the sex differences observed in cBMA.

Both cBMA and subacromial bursa tissue appear to be successful in forming colonies in culture. The results of this study suggest the time to form colonies may be less in subacromial bursa tissue than cBMA. There are similar numbers of colonies formed in the 2 tissue types, although more work must be done to elucidate this. The authors believe that the subacromial bursa may be a source of regenerative CTPs. Studies have demonstrated that the paratenon may be instrumental in patella tendon healing. ${ }^{27-29}$ The authors feel that the subacromial bursa may serve a similar role in rotator cuff regeneration despite being an extrinsic tissue, perhaps accounting for the large presence of CTPs.

Although CTPs have become a frequent topic of discussion in the literature, there is significant variability in quantification of progenitor cell "potency." Time to form colonies represents a novel and potentially useful metric for identifying CTP efficacy. Samsonraj et al. ${ }^{30}$ discuss the importance of colony-forming efficacy in cell lines with greater growth capacity. In a separate article, Samsonraj et al. ${ }^{19}$ proposed "population doubling time" as a marker for stem cell efficacy. Although the exact implications of the results presented here remain unknown, time to form colonies may prove to be a useful metric for measuring CTP viability, as supported by this work.

Improving healing potential following arthroscopic rotator cuff repair has recently garnered increased interest among shoulder surgeons. Thus, along with mechanical and technical failures, biologic failure may 
Days to Form Colonies: Bursa vs. cBMA

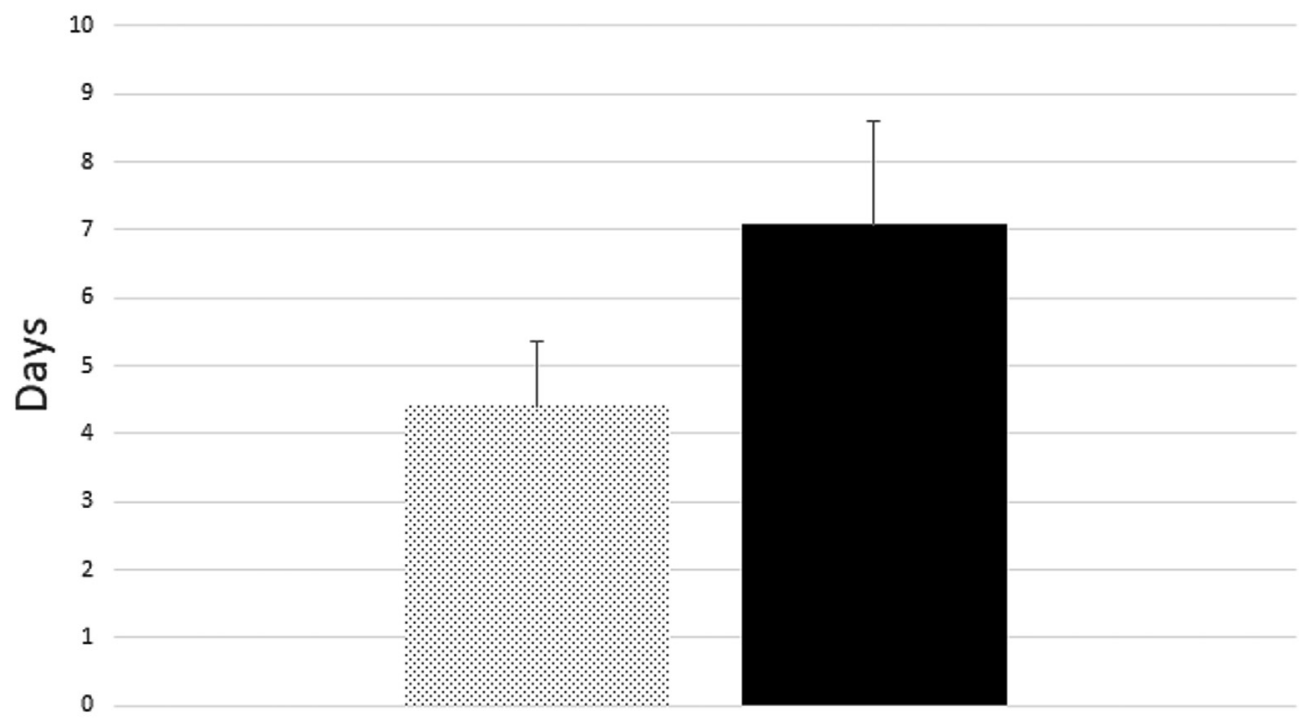

Fig 5. Average time to form colonies (days) for bursa and cBMA. (cBMA, concentrated bone marrow aspirate.)

: Bursa a cBMA

play an important role in rotator cuff retears. When considering patient age, Wagner et al. ${ }^{31}$ discuss the role of aging in endogenous CTPs as it relates to fracture healing, noting a significant decrease in viability, cell migration, and the number of CTPs in elderly patients. This notion is supported by work in murine models. ${ }^{32}$ The data presented in this study may help to guide provider-patient discussions regarding the usage of autologous CTP augmentation during rotator cuff repair. Older patients may prove to be less optimal candidates for this therapy. Future clinical trials will focus on the outcomes of therapy in various patient demographic cohorts and determine whether there is a threshold for time to form colonies that must be reached to render CTP augmentation useful to patients in vivo.

Despite the presence of available CTPs in multiple tissue types, cBMA has been the most prevalent choice of donor site within orthopaedic surgery. Gianakos et al. $^{8}$ describe the widespread use of concentrated

Total Colony Forming Units: Bursa vs. cBMA

Fig 6. Total colonies formed (CFU) for bursa and cBMA. (cBMA, concentrated bone marrow aspirate; CFU, colonyforming units.)
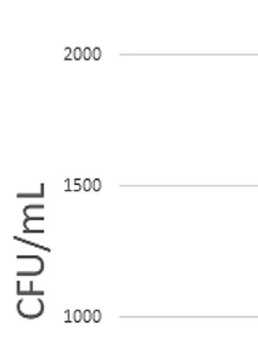

500
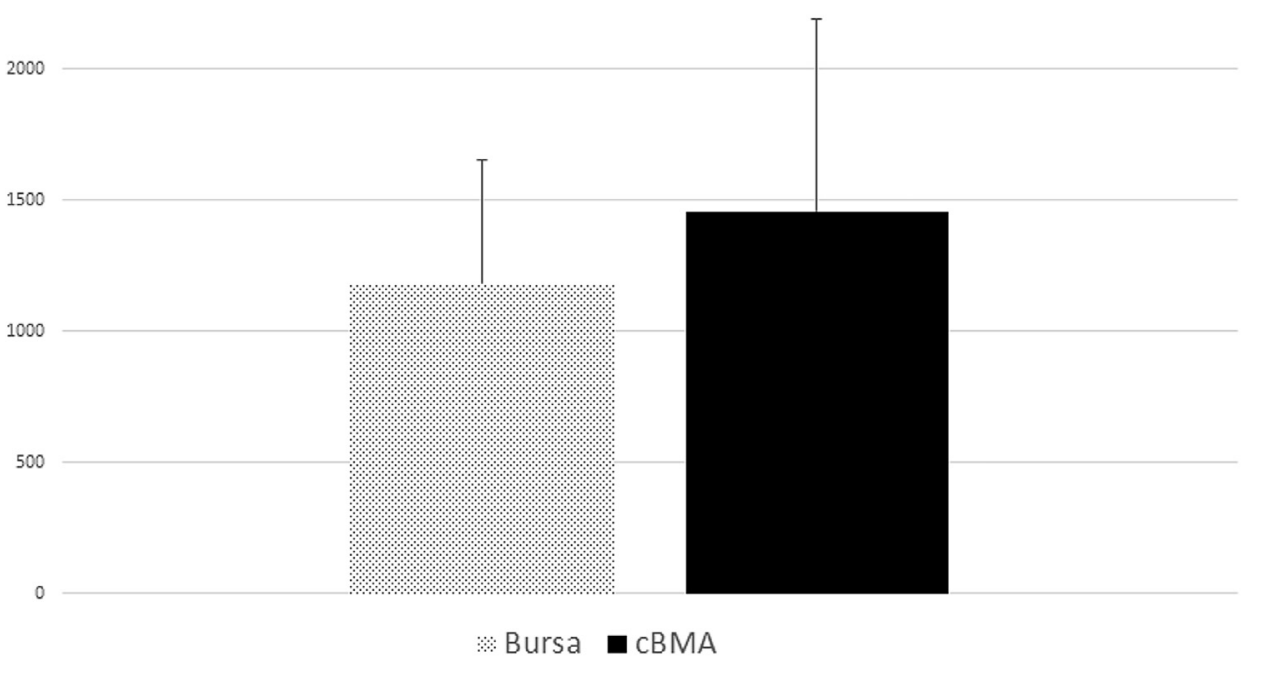
cBMA for cartilaginous lesions, bone defects, and tendon injuries described in the orthopaedic literature, as well as a wide variety of techniques for harvesting and preparing cBMA samples. Specifically, autologous cBMA for harvesting of BMCs has been described as a promising adjunct for patients undergoing rotator cuff repair to augment the healing process. ${ }^{2,33,34}$ Although this technology provides a potential avenue for improved healing, there is still variation within the literature regarding the success of supplementation with CTPs in these patients. ${ }^{35}$ More thorough analysis, such as what is described in this study, may help to identify which patients will best benefit from CTP augmentation of rotator cuff surgery.

CTPs have been identified within the subacromial bursa tissue. ${ }^{13-17,36}$ Given that subacromial bursa tissue is frequently discarded during rotator cuff surgery, this tissue presents an optimal choice of donor site for CTPs for use during arthroscopic rotator cuff repair. Previous work by Dyrna et al. ${ }^{28}$ has contributed that bursal donated cells may have superior engraftment and survival in healing tendon than cBMA-derived cells. The results of this study further confirm that bursa is capable of forming colony units in culture. Subacromial bursa tissue may represent a cost-effective method of CTP procurement. Further, there is minimal donor-site morbidity entailed in bursal harvesting, given that this is typically already part of the rotator cuff repair.

With better understanding of factors impacting CTP viability, providers will be able to more aptly target patients likely to have successful outcomes from autologous CTP therapy. Future work is necessary to determine necessary thresholds of time to form colonies of CTPs for success of these therapies and further validate the use of this metric.

The authors confirmed the hypothesis that older age was associated with increased time to form colonies in culture in both cBMA and subacromial bursa tissue. However, contrary to the hypothesis, increased initial cellularity of cBMA sample was not associated with more rapid colony formation. Time to form colonies in culture may prove to be an important, measurable variable in CTP functionality and efficacy. In addition, there are significant variations of in vitro characteristics of harvested CTPs based on patient characteristics, namely age. Subacromial bursa tissue represents a viable alternative to cBMA for autologous CTP harvest.

\section{Limitations}

Limitations of our study include the in vitro nature of the design. Clinical outcome trials will be necessary to repeat these results in vivo, with outcomes based on tendon healing, outcome scores, etc. In addition, there is inevitable variation in characteristics of samples based on intrinsic patient demographics, which the large sample size attempts to control for. Furthermore, there is known variability of cBMA in subacromial bursa tissue samples that may account for measured differences even within a single patient. Various preparation protocols, testing kits, and added chemical agents of cBMA and subacromial bursa tissue may impact the time to form colonies, limiting the generalizability of this data in outside institutions. Lastly, initial cellularity was only available for cBMA, and thus could not be compared to subacromial bursa tissue samples.

\section{Conclusions}

Increased age (in cBMA and subacromial bursa tissue) and lower initial cellularity (in cBMA) are associated with longer time to form colonies in culture.

\section{References}

1. Kingery MT, Manjunath AK, Anil U, Strauss EJ. Bone marrow mesenchymal stem cell therapy and related bone marrow-derived orthobiologic therapeutics. Curr Rev Musculoskelet Med 2019;12:451-459.

2. Hernigou P, Flouzat Lachaniette CH, Delambre J, et al. Biologic augmentation of rotator cuff repair with mesenchymal stem cells during arthroscopy improves healing and prevents further tears: A case-controlled study. Int Orthop 2014;38:1811-1818.

3. Ding DC, Shyu WC, Lin SZ. Mesenchymal stem cells. Cell Transplant 2011;20:5-14.

4. Almalki SG, Agrawal DK. Key transcription factors in the differentiation of mesenchymal stem cells. Differentiation 2016;92:41-51.

5. Ahmad Z, Wardale J, Brooks R, Henson F, Noorani A, Rushton N. Exploring the application of stem cells in tendon repair and regeneration. Arthroscopy 2012;28: 1018-1029.

6. Carr JB 2nd, Rodeo SA. The role of biologic agents in the management of common shoulder pathologies: Current state and future directions. J Shoulder Elbow Surg 2019;28: 2041-2052.

7. Centeno CJ, Al-Sayegh H, Bashir J, Goodyear S, Freeman MD. A prospective multi-site registry study of a specific protocol of autologous bone marrow concentrate for the treatment of shoulder rotator cuff tears and osteoarthritis. J Pain Res 2015;8:269-276.

8. Gianakos AL, Sun L, Patel JN, Adams DM, Liporace FA. Clinical application of concentrated bone marrow aspirate in orthopaedics: A systematic review. World $J$ Orthop 2017;8:491-506.

9. Murray IR, Robinson PG, West CC, et al. Reporting standards in clinical studies evaluating bone marrow aspirate concentrate: A systematic review. Arthroscopy 2018;34:1366-1375.

10. Beitzel K, McCarthy MB, Cote MP, et al. Comparison of mesenchymal stem cells (osteoprogenitors) harvested from proximal humerus and distal femur during arthroscopic surgery. Arthroscopy 2013;29:301-308.

11. Beitzel K, McCarthy MB, Cote MP, et al. Rapid isolation of human stem cells (connective progenitor cells) from the distal femur during arthroscopic knee surgery. Arthroscopy 2012;28:74-84. 
12. Voss A, McCarthy MB, Singh H, et al. The influence of trocar fenestration and volume on connective tissue progenitor cells (stem cells) in arthroscopic bone marrow aspiration from the proximal humerus. Arthroscopy 2017;33:1167-1174.e1161.

13. Steinert AF, Kunz M, Prager P, et al. Characterization of bursa subacromialis-derived mesenchymal stem cells. Stem Cell Res Ther 2015;6:114.

14. Steinert A, Heymer A, Kunz M, Jakob F, Noth U, Gohlke F. Adult multilineage mesenchymal progenitor cells isolated from the bursa subacromialis. J Stem Cells Regen Med 2007;2:188-189.

15. Utsunomiya H, Uchida S, Sekiya I, Sakai A, Moridera K, Nakamura T. Isolation and characterization of human mesenchymal stem cells derived from shoulder tissues involved in rotator cuff tears. Am J Sports Med 2013;41: 657-668.

16. Lhee SH, Jo YH, Kim BY, et al. Novel supplier of mesenchymal stem cell: subacromial bursa. Transplant Proc 2013;45:3118-3121.

17. Randelli P, Randelli F, Ragone V, et al. Regenerative medicine in rotator cuff injuries. Biomed Res Int 2014;2014: 129515.

18. Uhthoff HK, Sarkar K. Surgical repair of rotator cuff ruptures. The importance of the subacromial bursa. J Bone Joint Surg Br 1991;73:399-401.

19. Samsonraj RM, Raghunath M, Nurcombe V, Hui JH, van Wijnen AJ, Cool SM. Concise review: Multifaceted characterization of human mesenchymal stem cells for use in regenerative medicine. Stem Cells Transl Med 2017;6: 2173-2185.

20. Castro-Malaspina H, Gay RE, Resnick G, et al. Characterization of human bone marrow fibroblast colonyforming cells (CFU-F) and their progeny. Blood 1980;56: 289-301.

21. Dominici M, Le Blanc K, Mueller I, et al. Minimal criteria for defining multipotent mesenchymal stromal cells. The International Society for Cellular Therapy position statement. Cytotherapy 2006;8:315-317.

22. Patterson TE, Boehm C, Nakamoto C, et al. The efficiency of bone marrow aspiration for the harvest of connective tissue progenitors from the human iliac crest. J Bone Joint Surg Am 2017;99:1673-1682.

23. Nazal MR, McCarthy MBR, Mazzocca AD, Martin SD. Connective tissue progenitor analysis of bone marrow aspirate concentrate (BMAC) harvested from the body of the ilium during arthroscopic acetabular labral repair. Arthroscopy 2020;36:1311-1320.
24. Davies BM, Snelling SJB, Quek L, et al. Identifying the optimum source of mesenchymal stem cells for use in knee surgery. J Orthop Res 2017;35:1868-1875.

25. Guan YT, Xie Y, Li DS, et al. Comparison of biological characteristics of mesenchymal stem cells derived from the human umbilical cord and decidua parietalis. Mol Med Rep 2019;20:633-639.

26. Morikawa D, Muench LN, Baldino JB, et al. Comparison of preparation techniques for isolating subacromial bursaderived cells as a potential augment for rotator cuff repair. Arthroscopy 2020;36:80-85.

27. Dyment NA, Liu CF, Kazemi N, et al. The paratenon contributes to scleraxis-expressing cells during patellar tendon healing. PLoS One 2013;8:e59944.

28. Dyrna F, Zakko P, Pauzenberger L, McCarthy MB, Mazzocca AD, Dyment NA. Human subacromial bursal cells display superior engraftment versus bone marrow stromal cells in murine tendon repair. Am J Sports Med 2018;46:3511-3520.

29. Dyment NA, Hagiwara Y, Matthews BG, Li Y, Kalajzic I, Rowe DW. Lineage tracing of resident tendon progenitor cells during growth and natural healing. PLoS One 2014;9: e96113.

30. Samsonraj RM, Rai B, Sathiyanathan P, et al. Establishing criteria for human mesenchymal stem cell potency. Stem Cells 2015:33:1878-1891.

31. Wagner DR, Karnik S, Gunderson ZJ, et al. Dysfunctional stem and progenitor cells impair fracture healing with age. World J Stem Cells 2019;1 1:281-296.

32. Bruna F, Contador D, Conget P, Erranz B, Sossa CL, Arango-Rodriguez ML. Regenerative potential of mesenchymal stromal cells: Age-related changes. Stem Cells Int 2016;2016:1461648.

33. Ellera Gomes JL, da Silva RC, Silla LM, Abreu MR, Pellanda R. Conventional rotator cuff repair complemented by the aid of mononuclear autologous stem cells. Knee Surg Sports Traumatol Arthrosc 2012;20:373-377.

34. Mazzocca AD, McCarthy MB, Chowaniec D, et al. Bone marrow-derived mesenchymal stem cells obtained during arthroscopic rotator cuff repair surgery show potential for tendon cell differentiation after treatment with insulin. Arthroscopy $2011 ; 27: 1459-1471$.

35. Murthi AM, Lankachandra M. Technologies to augment rotator cuff repair. Orthop Clin North Am 2019;50:103-108.

36. Song N, Armstrong AD, Li F, Ouyang H, Niyibizi C. Multipotent mesenchymal stem cells from human subacromial bursa: Potential for cell based tendon tissue engineering. Tissue Eng Part A 2014;20:239-249. 